\title{
ESTUDO DO PROCESSO DE PURIFICAÇÃO DE EFLUENTES OLEOSOS DA INDÚS- TRIA DE PETRÓLEO
}

\author{
J. C. GOMES FILHO ${ }^{1}$, S. H. V. CARVALHO ${ }^{1 *}$, J. I. SOLETTI ${ }^{1}$, A. S. PEITER ${ }^{2}$, JOSÉ L. G. MA- \\ RINHO $^{1}$, L. MEILI $^{1}$ \\ ${ }^{1}$ Universidade Federal de Alagoas, Centro de Tecnologia \\ ${ }^{2}$ Universidade Federal de Alagoas, Unidade Santana do Ipanema \\ *e-mail: Sandra.ufal@gmail.com
}

\begin{abstract}
RESUMO
Derivados do petróleo, tais como óleos lubrificantes, gasolina, óleo diesel e seus compostos orgânicos constituintes, são poluentes de difícil degradação, constantemente liberados nas bacias hidrográficas, como resultado de diferentes atividades industriais, provocando sérios problemas de poluição à vida aquática. A crescente preocupação com o meio ambiente tem intensificado o estudo de técnicas de remoção destas cargas poluidoras dos efluentes. Neste sentido, a filtração tem se destacado, pois se apresenta como um método eficaz, simples e econômico. Tem sido intensificada a pesquisa por materiais filtrantes de baixo custo e reutilizáveis em outras funções, tendo seu valor agregado aumentado pelo processo de filtração. Uma vez que o bagaço de cana é abundante no país, possui elevada área superficial e é altamente hidrofóbico, seu uso como leito filtrante de contaminantes orgânicos tem despertado interesse da comunidade acadêmica. Este trabalho tem por objetivo o estudo de uma nova metodologia para remoção de teores de óleos e graxas, utilizando processo de filtração. Os resultados obtidos mostraram a eficácia do bagaço de cana como meio filtrante. A remoção do teor de óleos e graxas aumentou à medida que foram realizadas as corridas com o aumento da compactação do leito.
\end{abstract}




\section{INTRODUÇÃO}

A água é um dos recursos naturais mais utilizados. A indústria, através das atividades desenvolvidas em seu interior, representa um setor de atividade grande usuário de água. Dessa forma, carece estar atento aos meios disponíveis para se utilizar de forma eficiente esse recurso natural. Os usos da água na indústria podem ser divididos em um dos grupos a seguir: transferência de calor, geração de energia e aplicação a processos (DANTAS NETO, 2015).

$\mathrm{O}$ efluente gerado em terminais de distribuição de combustíveis terrestre geralmente tem uma elevada carga orgânica. Esta carga orgânica é difícil de ser degradada por microorganismos porque contém constituintes de hidrocarbonetos da gasolina e do óleo diesel (VIEIRA et al., 2012).

Efluentes são uma importante fonte de poluição ambiental aquático. Os efluentes são compostos de óleo e gordura juntamente com muitos outros compostos orgânicos tóxicos. Embora tenham sido feitos esforços para substituir os combustíveis fósseis, o petróleo continua a ser uma importante matéria-prima. A necessidade de satisfazer a crescente procura global de energia, que é esperada subir $44 \%$ ao longo das próximas duas décadass, faz do processamento de petróleo bruto e da geração de efluentes de refinaria de petróleo questões de importância global (DIYA'UDDEEN, DAUD e AZIZ, 2011).

Um dos maiores custos associados com o tratamento de efluentes é a importação de substâncias químicas para o tratamento de água e outros materiais. Uma solução para este problema é a produção local das substâncias químicas de tratamento, ou materiais não convencionais localmente disponíveis. Muitos adsorventes não convencionais de baixo custo têm sido experimentados por muitos pesquisadores, tais como resíduo de bioreator (bio- gás residual slurry), casca de banana, quitosana, bagaço de cana-de-açúcar, serragem de madeira, casca de semente de Moringa oleifera, fibra de coco e casca de laranja (NAMASIVAYAM et al, 2001).

O setor agrícola produz anualmente grandes quantidades de cana-de-açúcar e seu processamento industrial igualmente gera toneladas de bagaço. Bagaço de cana de açúcar é constituído por cerca de $50 \%$ de celulose, $25 \%$ de hemicelulose e $25 \%$ de lignina. Cerca de 54 milhões de toneladas secas de bagaço é produzido anualmente em todo o mundo (MOUBARIK e GRIMI, 2015).

Devido a esse panorama foi identificado que o bagaço de cana é um excelente meio filtrante, podendo ser uma boa alternativa para o tratamento de efluentes oleosos da indústria de petróleo. Nesse contesto este trabalho busca desenvolvimento e aprimoramento de uma metodologia para o processo de filtração utilizando como método uma coluna de filtração com bagaço de cana como meio filtrante.

\section{MATERIAIS E MÉTODOS}

A metodologia utilizada neste trabalho foi dividida em: preparo da unidade, preparo do efluente, funcionamento da coluna, realização do experimento, análise de Teor de Óleos e Graxa (TOG) no efluente e análise de TOG no meio filtrante.

\subsection{Preparo da Unidade}

Foi montada uma unidade de filtração para retenção das partículas oleosas no Laboratório de Sistemas de Separação e Otimização de Processos (LASSOP) constituída por: um tanque de alimentação de 200 litros, uma bomba centrífuga, uma peristáltica, um rotâmetro de vazão na linha de alimentação; um manômetro para gases instalado na célula de 
filtração; válvulas globo e a coluna filtrante (Figura 1).

Figura 1- Unidade filtrante

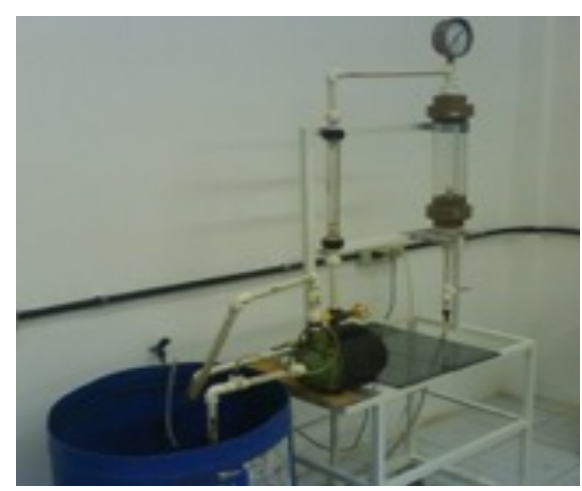

Fonte: Autor (2010).

\subsection{Preparo do Efluente}

O efluente sintético foi preparado na tentativa de simular o efluente real da indústria de petróleo. O petróleo cru foi adicionado em linha na coluna com uma vazão de $2 \mathrm{ml} / \mathrm{min}$ através de uma bomba peristáltica, que o manteve em circulação para uma maior homogeneização.

\subsection{Funcionamento da Coluna}

A coluna é operada em circuito aberto, o efluente é impulsionado através de uma bomba centrífuga para alimentar a coluna, onde o mesmo por sua vez entra em contato com o meio filtrante, bagaço de cana. Após atravessar todo meio filtrante o efluente é descartado pela base da coluna em um tanque de coleta.

\subsection{Realização do Experimento}

Foram realizadas 3 corridas experimentais. Na primeira corrida foi adicionado ao tanque de alimentação $75 \mathrm{~L}$ do efluente, e a coluna preenchida com o recheio (bagaço de cana) em cerca de $80 \%$ do seu volume, aproximadamente $200 \mathrm{~g}$ de bagaço. Acionou-se a bomba para iniciar a filtração onde a vazão de entrada foi ajustada e mantida constante a 200 $\mathrm{L} / \mathrm{h}$. As coletas do material filtrado foram realizadas após os tempos pré-determinados de 10,18 e $22 \mathrm{~min}$, para analise da remoção de óleos e graxas através do TOG.

Após 22min de operação o primeiro experimento foi encerrado.

Para a segunda corrida o tanque de alimentação foi preenchido com cerca de $155 \mathrm{~L}$ de efluente e o recheio da coluna foi mantido. Foi observado que houve uma redução do nível de recheio na coluna devido a sua compactação. Repetiu-se o procedimento semelhante à primeira corrida experimental, alterando apenas os tempos de coleta para 10, 20, 30, 40 e 46min.

Para a terceira corrida o tanque foi preenchido com cerca de $150 \mathrm{~L}$ de efluente e repetiu-se o procedimento anterior, a vazão de entrada caiu para 170 1/h devido à compactação do recheio. Os tempos de coletas foram 10, 20, 30, 40 e $50 \mathrm{~min}$

Ao final das três corridas o TOG, das amostras coletadas, e do recheio foi analisado.

\subsection{Analise de TOG No Efluente}

Para efetuar a análise, foi coletada inicialmente uma alíquota de $50 \mathrm{~cm}^{3}$ da amostra. À esta alíquota adicionou-se $10 \mathrm{~cm}^{3}$ de $\mathrm{n}$-hexano para extração do óleo. A seguir agitou-se a mistura e deixou-se em repouso, até que se formassem duas fases visualmente distintas. Uma fase é composta basicamente de água e a outra, a fase mais leve, de n-hexano e óleo. Após a separação das fases, retirou-se uma alíquota de $50 \mu \mathrm{L}$ da fase do n-hexano e óleo, e distribuiu-se sobre a lente do aparelho (TOG/TPH Analyzer da Infracal) da maneira mais homogênea possível e efetuou-se a leitura do TOG. A mesma é expressa em mg/L.

\subsection{Analise de TOG No Meio Filtrante}


As amostras de bagaço foram postas para secar em uma estufa a $90^{\circ} \mathrm{C}$, foram colocadas em cartuchos de papel de filtro. Foi medido o peso do material neles contidos. Os cartuchos foram colocados no interior do extrator (Soxhlet), e adaptados ao balão. Foi adcionado o solvente (n-hexano) ao balão e o aquecimento foi iniciado, após cerca de uma hora e meia todo óleo foi extraído. A massa da amostra foi calculada e subtraída da massa inicial para encontrar a massa de óleo contida na amostra.

\section{RESULTADOS E DISCUSSÃO}

A Tabela 1 apresenta os resultados obtidos na primeira corrida experimental, onde foram tratados aproximadamente $74 \mathrm{~L}$ de efluente. Foram coletadas 3 amostras $\left(\mathrm{A}_{1}, \mathrm{~A}_{2}\right.$ e $A_{3}$ ) da entrada e da saída da coluna e analisados TOG $_{\mathrm{E}}$ (TOG de entrada do efluente), TOGS (TOG de saída do efluente tratado) e calculado o TOGR (remoção de TOG após o tratamento) os tempos de coleta (TC) foram 10,18 e $22 \mathrm{~min}$.

Tabela 1 - Corrida 1 (Resultados de TOG)

$\begin{array}{ccccc}\text { Amostra } & \begin{array}{c}\mathrm{T}_{\mathrm{C}} \\ (\mathrm{min})\end{array} & \begin{array}{c}\mathrm{TOG}_{\mathrm{E}} \\ (\mathrm{ppm})\end{array} & \begin{array}{c}\mathrm{TOG}_{\mathrm{S}} \\ (\mathrm{ppm})\end{array} & \begin{array}{c}\mathrm{TOG}_{\mathrm{R}} \\ (\%)\end{array} \\ \mathrm{A}_{1} & 10 & 168,5 & 127,3 & 24,43 \\ \mathrm{~A}_{2} & 18 & 157,7 & 87,7 & 44,40 \\ \mathrm{~A}_{3} & 22 & 171,0 & 81,0 & 52,63\end{array}$

Fonte: Autor (2010).

A Tabela 2 foi construída com os resultados da segunda corrida experimental onde foram tratados cerca de $154 \mathrm{~L}$ de efluente o que permitiu um numero maior de amostras analisadas.
Tabela 2- Corrida 2 (Resultados de TOG)

\begin{tabular}{ccccc} 
Amostra & $\begin{array}{c}\text { TC } \\
(\mathrm{min})\end{array}$ & $\begin{array}{c}\mathrm{TOG}_{\mathrm{E}} \\
(\mathrm{ppm})\end{array}$ & $\begin{array}{c}\mathrm{TOG}_{\mathrm{S}} \\
(\mathrm{ppm})\end{array}$ & $\begin{array}{c}\mathrm{TOG}_{\mathrm{R}} \\
(\%)\end{array}$ \\
\hline $\mathrm{A}_{4}$ & 10 & 145,7 & 53,7 & 63,16 \\
$\mathrm{~A}_{5}$ & 20 & 159,8 & 54,7 & 65,79 \\
$\mathrm{~A}_{6}$ & 30 & 148,3 & 37,4 & 74,78 \\
$\mathrm{~A}_{7}$ & 40 & 150,7 & 36,5 & 75,77 \\
$\mathrm{~A}_{8}$ & 46 & 157,0 & 30,3 & 80,70
\end{tabular}

Fonte: Autor (2010).

$\mathrm{Na}$ terceira e última corrida experimental o volume de efluente tratado foi aproximadamente $142 \mathrm{~L}$. Os resultados obtidos foram inseridos na tabela 3 .

Tabela 3- Corrida 3 (Resultados de TOG)

$\begin{array}{ccccc}\text { Amostra } & \begin{array}{c}\mathrm{T}_{\mathrm{C}} \\ (\mathrm{min})\end{array} & \begin{array}{c}\mathrm{TOG}_{\mathrm{E}} \\ (\mathrm{ppm})\end{array} & \begin{array}{c}\mathrm{TOG}_{\mathrm{S}} \\ (\mathrm{ppm})\end{array} & \begin{array}{c}\mathrm{TOG}_{\mathrm{R}} \\ (\%)\end{array} \\ \mathrm{A}_{9} & 10 & 196,3 & 26,0 & 86,76 \\ \mathrm{~A}_{10} & 20 & 202,7 & 21,3 & 89,47 \\ \mathrm{~A}_{11} & 30 & 197,3 & 16,3 & 91,72 \\ \mathrm{~A}_{12} & 40 & 209,0 & 17,0 & 91,87 \\ \mathrm{~A}_{13} & 50 & 198,3 & 14,7 & 92,61\end{array}$

Fonte: Autor (2010).

A partir dos dados das tabelas 1, 2 e 3 foi observado que ao decorrer das corridas a remoção do TOG aumentou de forma significativa, isso se deve ao fato da compactação do leito filtrante. A compactação do leito gerou 
um aumento na pressão interna da coluna e uma diminuição na vazão de entrada da mesma. Os dados da Tabela 4 mostram mais detalhadamente as diferenças operacionais entre as corridas experimentais. A vazão de entrada (Q) de efluente teve uma redução devido à compactação do leito, isso também provocou um aumento na pressão interna da coluna (PC) os volumes (V) de efluente tratados foram preparados para cada corrida experimental, mas o leito foi mantido nas 3 corridas.

Tabela 4- Variáveis operacionais

\begin{tabular}{ccccc} 
Corrida & $\begin{array}{c}\mathrm{Q} \\
(\mathrm{L} / \mathrm{h})\end{array}$ & $\begin{array}{c}\mathrm{V} \\
(\mathrm{L})\end{array}$ & $\begin{array}{c}\mathrm{P}_{\mathrm{C}}(\mathrm{Kgf} / \\
\left.\mathrm{cm}^{2}\right)\end{array}$ & $\begin{array}{c}\mathrm{TOG}_{\mathrm{R}} \\
(\%)\end{array}$ \\
\hline 1 & 200 & 73,3 & 0,4 & 52,6 \\
2 & 200 & 153,3 & 0,6 & 80,7 \\
3 & 170 & 141,7 & 0,9 & 92,6
\end{tabular}

Fonte: Autor (2010).

Foi possível observar que o próprio óleo retido no leito filtrante funcionou como um filtro natural, já que o óleo concentrado acabou retendo as partículas diluídas do efluente.

Na Figura 2 pode-se observar que a remoção do TOG aumentou à medida que foram realizadas as corridas com o aumento da compactação do leito.

Figura 2 - Remoção de TOG

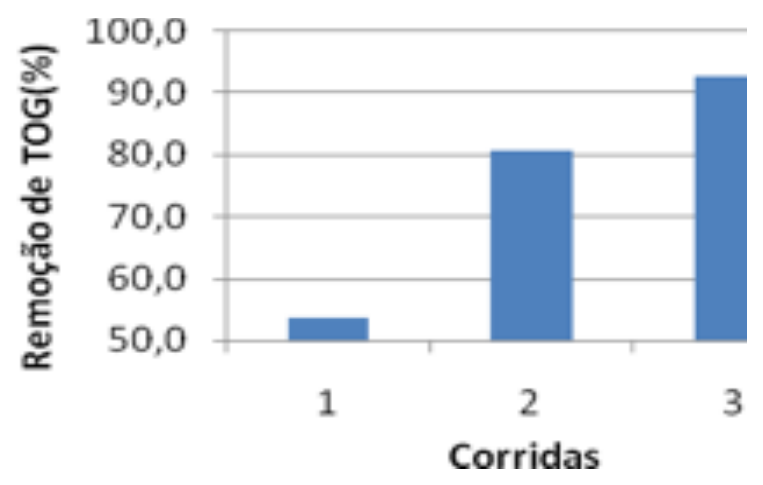

Fonte: Autor (2010).

A Figura 3 mostra as alturas onde foram coletadas as amostras de bagaço.

Figura 3 - Coluna filtrante

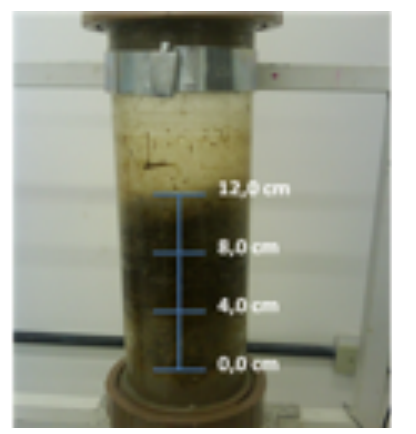

Fonte: Autor (2010).

A Tabela 5 apresenta os dados das análises do óleo que ficou retido em cada camada analisada. As massas das amostras de bagaço (mbagaço $)$ e as massas de óleo retidas nas amostras (móleo) foram calculadas para encontrar a porcentagem de remoção do bagaço em cada camada de recheio.

Tabela 5 - Óleo retido no recheio

\begin{tabular}{cccc}
$\begin{array}{c}\text { Altura } \\
\text { recheio }(\mathrm{cm})\end{array}$ & $\begin{array}{c}\text { mbagaço } \\
(\mathrm{g})\end{array}$ & $\begin{array}{c}\text { móleo } \\
(\mathrm{g})\end{array}$ & $\begin{array}{c}\text { móleo } \\
(\%)\end{array}$ \\
\hline 8 a 12 & 3,865 & 1,114 & 22,38 \\
4 a 8 & 5,830 & 0,649 & 10,03 \\
0 a 4 & 4,980 & 0,125 & 2,46
\end{tabular}

Fonte: Autor (2010).

Os dados da tabela mostram que a remoção de óleo do efluente foi significativamente maior nos níveis mais altos da coluna, isso poder ser explicado pelo fato de que a parte mais alta tem o primeiro contato com efluente com concentrações elevadas de óleo e o próprio óleo retido na parte superior ajuda na remoção. 


\section{CONCLUSÃO}

Pelos resultados obtidos pode-se afirmar que o bagaço de cana é um excelente biosorvente de óleo onde se constatou até $92 \%$ de remoção do teor de óleos e graxas mesmo com o leito parcialmente saturado de óleo.

O bagaço saturado de óleo pode ser usado como combustível, possuindo um poder calorífico mais elevado do que o mesmo sem o óleo.

\section{REFERÊNCIAS}

DANTAS NETO, J. Uso eficiente da água: aspectos teóricos e práticos. Disponível em: http:/www.eumed.net/libros-gratis/ 2008c/447/USO \%20EFICIENTE\%20DA $\% 20 A G U A \% 20 \mathrm{NA} \% 20 I N D U S T R I A . h t m$. Acesso em: 15 mar de 2015.

DIYA'UDDEEN, B. H.; DAUD, W. M. A. W.; AZIZ, A. R. A. Treatment technologies for petroleum refinery effluents: A review. Process Safety and Environmental Protection, Volume 89, Issue 2, Pages 95-105, 2011.

MOUBARIK, A.; GRIMI, N. Valorization of olive stone and sugar cane bagasse byproducts as biosorbents for the removal of cadmium from aqueous solution. Food Research International, volume 73, pg.169175, 2015.

NAMASIVAYAM, C.; KUMAR, M. D.; SELVI, K.; BEGUM, R.A.; VANATHI, T.; YAMUNA, R. T. Waste Coir Pith - a Potencial Biomass for the Treatment of Dyeing Wastewaters. Biomass \& Bioenergy, n. 21, p. 477-483, 2001.

VIEIRA,R. B.; VIEIRA, P. A.; CARDOSO, S. L.; RIBEIRO, E. J.; CARDOSO, V. L. Sedimentation of mixed cultures using natural coagulants for the treatment of effluents generated in terrestrial fuel distribution terminals. Journal of Hazardous Materials, Volume 231-232, Issue null, Pages 98-104, 2012. 